\title{
La Evaluación a través de Portafolios: ¿Una Ocasión para el Aprendizaje?
}

\author{
The Assessment Trough Portfolios: An Opportunity for \\ Learning?
}

\author{
Noelia Alcaraz Salarirche* \\ Universidad de Cádiz
}

\begin{abstract}
Autores como Calfee y Perfumo (1993), Shores y Grace (2004), Delamstro (2005), Barberá (2005), Darling-Hammond (2005) o Pérez Rendón (2014) defienden un uso del portafolios centrado en el aprendizaje del-la estudiante y resaltan la vertiente reflexiva del mismo como una de las condiciones claves de la enseñanza y la evaluación a través de portafolios.

En este trabajo se exponen aquellas otras condiciones educativas -relacionadas con la reflexión sobre el aprendizaje y la enseñanza- que se han de tener en cuenta al poner en marcha un sistema de evaluación a través de portafolios.

Antes, se hace también un breve recorrido por el origen del portafolios, así como por los principales tipos, usos y definiciones que se encuentran del mismo.

El objetivo de este artículo es situar al lector ante un nuevo escenario de evaluación y brindar posibilidades alternativas de pensar la evaluación en un aula. Evaluación no confundida con la calificación, sino como proceso de comprensión permanente que busca la mejora tanto de la enseñanza como del aprendizaje.
\end{abstract}

Palabras claves: Evaluación, Portafolios, Reflexión, Retroalimentación, Enseñanza, Aprendizaje.

Authors like Calfee and Perfumo (1993), Shores and Grace (2004), Delamstro (2005), Barber (2005), Darling-Hammond (2005), or Pérez Rendón (2014) advocate a use of portfolio focused on the students' learning and highlight the reflective side of it as one of the key conditions of teaching and assessment through portfolios.

This paper shows those other educational conditions -related to reflection on learning and teaching- which should be taken into account when implementing a system of assessment through portfolios.

Before, the work provides an overview of the origin of the portfolio, as well as its main types, uses and definitions.

The aim of this article is to place the reader in a new assessment scenario and provide alternative possibilities of thinking about classroom assessment. This assessment should not be confused with a grade (US) / mark (UK), but seen as a permanent understanding process that seeks to improve both teaching and learning.

Keywords: Evaluation, Assessment, Portfolio, Reflection, Feedback, Teaching, Learning.

*Contacto: noelia.alcaraz@uca.es

issn: 1989-0397

www.rinace.net/riee/

https://revistas.uam.es/riee
Recibido:

$1^{\text {a }}$ Evaluación:

Aceptado:
24 de septiembre de 2015

25 de octubre de 2015

1 de diciembre de 2015 


\section{Introducción ${ }^{1}$}

El portafolios como herramienta para evaluar el proceso de aprendizaje del alumnado que cursa la etapa obligatoria de la enseñanza es una cuestión que resulta relativamente novedosa. Ello se debe a su escasa presencia y corta tradición en el ámbito educativo ya que este cuenta con una mayor trayectoria en contextos profesionales como los artísticos. Si bien es cierto que el uso del portafolios ha proliferado especialmente en los niveles de educación superior, el portafolios en su sentido literal, de 'portar folios', no es tan reciente. Lo novedoso del asunto sería otra cuestión: el abandono literal de la palabra, que convierte al portafolios en una práctica educativa al servicio de la evaluación y del aprendizaje. Es por ello que, como veremos a lo largo de este artículo, para que el portafolios sea algo más que un contenedor de trabajos de estudiantes que sirven para mostrar y demostrar las capacidades o competencias que estos poseen, se deben establecer una serie de condiciones que las prácticas con portafolios deben cumplir, siempre que estas pretendan estar al servicio de la evaluación -evaluación en el sentido de búsqueda de la mejora-. Es el reto ante el que nos encontramos: apostar por el diseño de un nuevo sistema de evaluación basado en la reflexión, la comunicación, la comprensión del error y el análisis de los procesos y contextos de aprendizaje. Se trata de una oportunidad para aprovechar la escasa tradición de prácticas con portafolios en las escuelas, y el carácter continuo y reflexivo que se le otorga en la literatura, para alejarnos del uso acreditativo y selectivo que este ha tenido en otros ámbitos.

\section{Breve recorrido por el origen, los tipos y los usos del portafolios}

\subsection{El nacimiento del portafolios en el ámbito educativo}

Históricamente el uso de los portafolios se sitúa en contextos profesionales relacionados sobre todo con las artes, la publicidad o las finanzas. Así lo exponen Danyelson y Abrutyn (1999): "Los portafolios, desde luego, no son una invención reciente. El concepto existe desde hace mucho en numerosos ámbitos fuera del aula. Artistas, arquitectos y fotógrafos lo usan para presentar su trabajo a clientes potenciales" (pp. 7-8).

En estos contextos los portafolios profesionales tenían y tienen como finalidad mostrar las mejores producciones elaboradas a lo largo de una determinada trayectoria profesional, debiendo cumplir con las demandas de intereses de los clientes a los que han de ser mostrados.

En palabras de Oosterhof (1996):

En esas profesiones, una persona compone un portafolios que contiene ejemplos de trabajo que se muestran a los clientes potenciales y a los empleadores. Los portafolios incluyen

\footnotetext{
${ }^{1}$ El contenido del presente artículo forma parte de la Tesis Doctoral de la autora, dirigida por Ángel I. Pérez Gómez, en el Departamento de Didáctica y Organización Escolar de la Facultad de Ciencias de la Educación, de la Universidad de Málaga.
} 
generalmente el mejor trabajo. Por otra parte, el contenido del portafolios también debe responder a los intereses y necesidades del cliente o empleador. (p. 165)

En este sentido, el uso que desde varios campos profesionales se ha venido dando al portafolios hace que este se presente como la vía a través de la cual profesionales o futuros profesionales debían demostrar sus conocimientos, habilidades y competencias en un determinado ámbito profesional, para ser valorado por el destinatario de dicho portafolios.

Es en la década de los noventa cuando en Europa empieza a extenderse hacia el ámbito educativo el interés por el portafolios. Los noventa, sobre todo a partir de su tercera década, se caracterizan, de acuerdo con Alcaraz (2015a), por la aparición de las ideas de Tyler, cuya intención era superar la mera evaluación psicológica. Es decir, el interés por el portafolios en el campo de la educación se sitúa especialmente en EE. UU. y Reino Unido, cuando empieza a surgir cierto descontento con las pruebas estandarizadas tipo test que tan a la orden del día estaban.

Este desarrollo se produce principalmente en el ámbito de la lectoescritura (en su estudio como lengua materna o como segunda lengua). Según Oosterhof (1996), una de las ventajas del portafolios con respecto a lo que hasta el momento existía -refiriéndose a las tradicionales pruebas de desempeño académico- era su fácil adaptabilidad a las metas educativas de cada estudiante. Sostenía que las pruebas escritas a menudo implicaban un conjunto común de ítems administrados simultáneamente a un grupo de estudiantes, mientras que "los portafolios, sin embargo, pueden ser totalmente individualizados. Cada portafolios puede ser sensible a los objetivos establecidos por cada estudiante" (Oosterhof, 1996, p. 166).

Por su parte, Delmastro (2005), que también sitúa el desarrollo histórico del portafolios y su mayor proliferación hacia mediados de los noventa en EE. UU. y Canadá, sostiene que es en los setenta cuando la UNESCO, con sus programas a nivel internacional para el desarrollo de la lectoescritura, comienza a provocar un mayor auge y difusión del portafolios como estrategia de evaluación en países del hemisferio occidental. La autora recoge que la mayoría de las investigaciones realizadas en ese ámbito contemplan la enseñanza y evaluación de destrezas de lectoescritura en el contex to del aprendizaje y desarrollo de la lengua materna (Hamilton, 1994; Jalbert, 1998; Murphy y Camp, 1996; Wile y Tierney, 1996; citados por Delmastro, 2005). Así mismo, Bastidas (1996), Reckase (1995) y Delmastro (2005) exponen que el uso del portafolios se ha ido extendiendo hacia Israel, Francia, Italia y Australia, lográndose avances en la incorporación del portafolios en educación desde el ámbito institucional, incluso llegándose a explorar sus posibilidades en la evaluación a gran escala en instituciones de EE. UU. y Canadá.

En este sentido, French (1992) centra la atención en el portafolios utilizado para atender a estudiantes de grupos minoritarios que aprenden el inglés como segunda lengua, que no son valorados de manera correcta por pruebas estandarizadas que no responden a sus características específicas.

Por otro lado, Lyons (1999) sitúa el origen del portafolios en la insatisfacción que existía con respecto a las escuelas norteamericanas en las décadas de los setenta y ochenta. De modo que la experimentación con portafolios parecía responder a la necesidad de implementar innovaciones focalizando la atención en docentes y estudiantes, inicialmente en relación con la evaluación y de manera más reciente vinculada a la formación de docentes. 
La consecuencia de todo ello es la proliferación en EE. UU. de multitud de experiencias escolares que introducen la práctica del portafolios como herramienta alternativa de evaluación. Surgen estudios, investigaciones y publicaciones sobre el tema en cuestión que tratan de documentar los procesos de implementación del portafolios en la enseñanza obligatoria. Rober C. Calfee y Pam Perfumo publican en 1993 en una revista llamada Journal of Reading un artículo titulado "Student Portfolios: Opportunities for a Revolution in Assessment" ("El Portafolios de Estudiante: Oportunidades Para una Revolución en Evaluación”). En él realizan un análisis del portafolios a partir de una investigación en la que diferentes centros hacen uso de este nuevo sistema de carpetas. Esto provocó que especialistas en psicometría se mostrasen inquietos ante tales acontecimentos, ya que la principal preocupación se centraba en cómo arbitrar mecanismos que permitiesen la estandarización del rendimiento y que cumpliesen con criterios de fiabilidad y validez. Sin embargo, eran los propios enseñantes quienes empezaban a tomar la iniciativa en el movimiento de sustitución de las pruebas estandarizadas (en parte o en su totalidad) por portafolios (Pelavin, 1991, citado por Calfee y Perfumo, 1993).

En España, no es hasta finales del siglo XX y principios del XXI cuando se empiezan a vislumbrar experiencias escolares que ponen en práctica el uso del portafolios. Contamos por tanto con una corta experiencia en el desarrollo de estas, especialmente en la educación obligatoria. Sin embargo, es cada vez más frecuente encontrarnos con experiencias de portafolios o de e-portafolios, sobre todo en el ámbito de la educación superior, de modo que la literatura educativa española que versa sobre el portafolios también comienza a proliferar.

Así por ejemplo, para Barberá (2005), el interés por el portafolios surge de un creciente interés por la elaboración de instrumentos y materiales útiles, acordes a los nuevos retos educativos. Según Wiggins (1990), la aparición de esta nueva práctica es un indicador de la búsqueda de mejoras en el contexto educativo, pretendiendo subsanar algunas importantes deficiencias en los sistemas educativos tradicionales más inmovilistas (citado por Barberá, 2005). Otros autores y autoras como DarlingHammond (2005), Díaz Barriga (2006), Monereo (2009) y Díaz Barriga y Pérez (2010) sostienen que la revisión de los portafolios, en los niveles superiores, se centra específicamente en los portafolios como instrumentos de "evaluación auténtica" de las competencias de los-as docentes. Díaz Barriga y Pérez (2010, p. 7) reconocen que "la entrada del discurso de las competencias en educación también ha resultado un factor clave que ha impulsado el interés por la evaluación mediante portafolios" (citado por Pérez Rendón, 2014, p. 26).

\subsection{Definiciones y tipos de portafolios}

Hace décadas que maestros y maestras incluyen en la lista del material escolar que se envía a las familias la conocida carpeta con gomillas. La idea, especialmente en los cursos inferiores, era que el niño o la niña incluyera en su carpeta cada uno de los trabajos que se iban realizando durante los tres trimestres del curso escolar para ser exhibidos a los familiares.

También en cursos superiores se ha conocido su existencia. La "carpeta”, "dossier", "carpeta memoria" o "portafolios" se ha venido usando como contenedor-organizador del material trabajado por el alumnado.

En este sentido, el portafolios ya existía y era entendido como un archivador que contenía el material escolar del alumnado. Se trata, por tanto, de una concepción 
completamente estática del portafolios que, poco a poco, va tratando de superarse en un intento por abandonar el sentido literal de este, como mero contenedor de los materiales producidos. Empieza a introducirse un nuevo componente que se convierte en uno de los elementos centrales de la literatura del portafolios: la implicación activa del alumnado en su propio proceso de aprendizaje a través de la reflexión conjunta con el profesor o la profesora.

Empieza a cuestionarse el sentido del portafolios como una acumulación simple de trabajos (García Hernández, 2000), sin propósitos referidos a la mejora del aprendizaje o de la práctica docente. En la formación inicial de profesorado cobra especial protagonismo en el prácticum, sirviendo a la reconstrucción y reelaboración del conocimiento práctico (Hidalgo y De la Blanca, 2013). Es decir, el término portafolios abandona su significado literal y comienza a asociarse a una serie de connotaciones más educativas relacionadas con los procesos de reflexión, de aprendizaje, de retroalimentación, de colaboración, de participación y de diálogo, entre otros, incorporándose así al lenguaje educativo.

Así, para Pollari (2000), se trata de un portafolios "cuando el alumno participa en ello en lugar de ser objeto evaluación” (Paulson, Paulson y Meyer, 1991, citado por Pollari, 2000, p. 18). El autor resalta el sentido del portafolios en tanto en cuanto favorece la autogestión y autorregulación del alumnado.

Un portafolios cuenta una historia. Es la historia de conocimiento. Conocimiento sobre cosas. Conocimiento sobre uno mismo. Conocimiento sobre la audiencia. Los portafolios son las propias historias de los estudiantes, de lo que ellos saben, del por qué ellos creen que lo saben y por qué otros podrían ser de la misma opinión. Un portafolios es una opinión construida. Los estudiantes demuestran lo que conocen con ejemplos de su trabajo. (Paulson y Paulson, 1991, p. 2)

Shores y Grace (2004) resaltan como objetivo último de la aplicación de portafolios el apoyo a la educación centrada en el alumnado. "El portafolios puede convertirse en el contexto en el que el niño o la niña reflexione sobre las ideas o conocimientos [...]. Además, pueden reflexionar sobre su progresión” (2004, pp. 29-30).

Para Villalobos, "la reflexión es lo que diferencia a los portafolios de las carpetas sobre escritura $[\ldots]$. En parte es a través de la reflexión que las carpetas o recopilaciones se transforman en portafolios" (2002, p. 391).

Pos su parte, Pérez Gómez entiende que "el portafolios se convierte en un punto de apoyo fundamental para la autoevaluación” (2012, p. 238). En este sentido coinciden Calero y Calero (2008), que fundamentan el uso del portafolios sobre la base del constructivismo. Para ellos, este puede llegar a ser un poderoso y flexible instrumento que está:

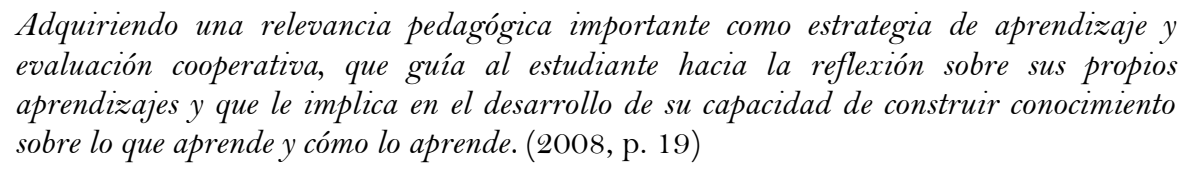

Adquiriendo una relevancia pedagógica importante como estrategia de aprendizaje y evaluación cooperativa, que guía al estudiante hacia la reflexión sobre sus propios aprendizajes y que le implica en el desarrollo de su capacidad de construir conocimiento sobre lo que aprende y cómo lo aprende. (2008, p. 19)

En definitiva, estas y otras definiciones parecidas muestran cómo el portafolios desde su aparición hasta la actualidad ha ido transformando su significado, despegándose cada vez más del sentido literal que tenía hasta ir incorporando un significado con connotaciones mucho más educativas. No obstante, el sentido y significado de un portafolios concreto dependerá del tipo de portafolios que estemos implementando, lo cual a su vez estará condicionado por el uso que la persona que lo pone en marcha pretende hacer de él. 
Si se hace un recorrido por la literatura que versa sobre portafolios, es fácil apreciar que existen variados tipos de portafolios. Por ejemplo, en el ámbito universitário, donde tienen una mayor presencia, se pueden encontrar prácticas con portafolios en diferentes ramas del saber como son las ciencias de la salud, ciencias experimentales, ingenierías, artes, humanidades y ciencias sociales, unas con mayor carga pedagógica y otras con menor. De modo que la variedad es amplia. Recojamos de Meeus y Lois (2006), citados por Pérez, Alcaraz y Fernández (2016), algunas de las posibles clasificaciones de portafolios: portafolios de presentación, portafolios de trabajo, portafolios para la retroalimentación, portafolios de rendición de cuentas, portafolios de producto, portafolios de evaluación, portafolios de crecimiento profesional y portafolios de proceso, entre otros ${ }^{2}$.

Así, se puede encontrar una amplia tipología de portafolios. Meeus y Lois (2006) distinguen hasta veintiocho clasificaciones de portafolios. Algunas de ellas se hacen en función del autor: discente o docente; del registro: papel o digital; de la defensa: escrito u oral; y del propósito: para la certificación, para la selección o para la promoción. Otra clasificación se hace en función del contenido, del momento de desarrollo del portafolios y de los objetivos que se persiguen (Barragán, 2005, citado por Pérez, Alcaraz y Fernández, 2016; Starkie, 2009).

De ahí que Barberá (2005) exponga que: "en la bibliografía encontraremos tantas definiciones y desarrollos de portafolios como autores seamos capaces de consultar (p. 499, citado por Pérez, Alcaraz y Fernández, 2016).

En este trabajo trataremos de resumir las aportaciones revisadas en dos categorías generales teniendo en cuenta que no se trata de una escala de negro o blanco, sino que también podemos encontrar matices.

La primera categoría, el primer tipo de portafolios que destacaremos, será el portafolios de producto (traducido como showcase) ${ }^{\text {s }}$. La segunda categoría o tipo sería el portafolios de proceso.

En el de producto, showcase portfolio, deja de prevalecer, y así lo expone Pollari (2000), el proceso. Lo que importa es el resultado final que se ha de exponer a una determinada audiencia. En el otro, en el de proceso, cobra protagonismo todo el proceso de enseñanza y aprendizaje, que trata de recogerlo pero que debe ir acompañado siempre de la comprensión, la reflexión conjunta y la autoevaluación del sujeto que aprende. Sin embargo, tanto uno como otro suelen usarse para obtener un mérito final, lo cual nos lleva a diferenciar los tipos pero a mezclar las funciones.

\subsection{Algunos usos del portafolios}

Pérez, Alcaraz y Fernández (2016) recogen la lista que Klenowski (2004) expone con los diferentes usos que se pueden encontrar del portafolios en el ámbito educativo:

\footnotetext{
2 Además de estas clasificaciones, hay que mencionar dos categorías más de portafolios que dependen del soporte: papel o virtual. El uso del e-portafolios ha proliferado mucho en la última década. Para profundizar en este, puede acudirse a Hernández (2008), Barret (2009) y Soto (2016), entre otros.
}

${ }^{3}$ Término que los anglosajones utilizan para referirse al portafolios de producto. 
$\checkmark$ El portafolios para la valoración del rendimiento, lo que denomina evaluación sumativa. Debe entenderse como calificación. Este acumula el material recogido durante un periodo de tiempo determinado.

$\checkmark$ El portafolios para conseguir una certificación o para la selección de profesionales es aquel que contiene una selección de los trabajos más representativos del aspirante.

$\checkmark$ El portafolios que permite promocionar dentro de un ámbito profesional. En función de los criterios de valoración expuestos por el contratante, el sujeto que aspira a ser promocionado selecciona los trabajos más acordes a tales criterios.

$\checkmark \quad$ El portafolios para el desarrollo profesional. Se refiere al portafolios de los-as docentes, cuyo fin es reflexionar críticamente sobre la propia práctica.

$\checkmark$ Por último, el portafolios para la evaluación y el aprendizaje, cuyo objetivo es ayudar al-la estudiante a desarrollar y profundizar sobre su proceso de aprendizaje.

Si profundizamos en las finalidades de cada uno, descubriremos que, de todos los posibles usos enumerados, solo uno de ellos no está asociado a la rendición de cuentas. Nos referimos al portafolios para el desarrollo profesional o, lo que es lo mismo, el portafolios del-la docente. El resto, incluido el portafolios para la evaluación y el aprendizaje, tiene un objetivo final que es demostrar algo. Con lo cual podríamos hablar de dos usos fundamentales: para rendir cuentas y para el desarrollo personal o profesional, en definitiva, para el aprendizaje.

\section{Condiciones de un portafolios para la evaluación y el aprendizaje}

Dada la relativa novedad del uso de portafolios en el ámbito educativo -a la que venimos haciendo referencia a lo largo del artículo-, nos encontramos ante el reto de tratar de dotarlo de sentido educativo, situándolo al servicio de la evaluación. Es decir, desplazar la función sumativa del mismo y resaltar la formativa.

Veremos a continuación las características o condiciones que un portafolios educativo debe tener o cumplir para convertirlo en esa "ocasión de aprendizaje" a la que se refería Álvarez Méndez (2008). O lo que es lo mismo, poner a la evaluación al servicio del aprendizaje para lo cual es condición necesaria, tal y como expone Alcaraz (2014), que esta fuese respetuosa con los principios psicológicos y pedagógicos que sustentan la visión constructivista del currículum y del aprendizaje en el aula, alejándose de la concepción tecnocrática de la enseñanza y suponiendo fuente de mejora para quien aprende y para quien enseña.

\subsection{El portafolios debe centrarse en el análisis y reflexión de los procesos y contextos de aprendizaje}

Tal y como defienden Román y Murillo (2009); Pérez Gómez, Soto, Sola y Serván (2009); Álvarez Méndez (2001); Alcaraz, Fernández y Sola (2012); Murillo e Hidalgo (2015), entre otros, la evaluación ha de servir para la mejora del aprendizaje del alumnado, es decir, la evaluación es también aprender cómo aprender. Pérez Gómez, Soto, Sola y Serván (2009) exponen:

De requisito de control burocrático, mecánico y externo que sanciona los rendimientos, establece calificaciones y clasifica a los estudiantes se convierte en un proceso complejo, cualitativo, de conocimiento de los propios procesos de aprendizaje, reflexión sobre ellos y 
planificación individual y compartida de proyectos de mejora, donde al final la evaluación incorporada como una cultura se convierte en el mejor instrumento de aprendizaje mediante la reflexión en la acción y sobre la acción. (209, p. 6)

Es a lo que Murillo e Hidalgo (2015) se refieren cuando expresan que una evaluación para la justicia social es aquella que, entre otras cuestiones, pasa de ser una evaluación "del" aprendizaje a una evaluación "para" el aprendizaje. De modo que desde esa perspectiva el portafolios debe servir "para" el aprendizaje; para auto-gestionar el propio proceso de aprendizaje, puesto que quien aprende evalúa a su vez, discrimina, valora, critica, opina, razona, fundamenta y decide entre lo que tiene valor y lo que no para el proceso de aprender. Por ello, el-la docente que pone en práctica la evaluación a través de portafolios debe preocuparse por incluir procesos de organización, de autoevaluación, de metacognición y de reflexión. Hebert (2001), Barberá (2005), Klenowsky (2004), Barret (2001), Lyons (1999) y Soto (2016), consideran a este último el elemento clave en el trabajo con portafolios.

De acuerdo con Soto (2016), la reflexión no se da de forma espontánea, sino que necesita aprenderse. Para Soto (2016) y Le Boterf (2000), la reflexión implica que la persona se distancie de su actividad para poder contemplar el problema y sus variables tratando de descontaminarse de su inmediatez. A ello es a lo que denominan metacognición.

La reflexión sobre los procesos y contextos en los que tiene lugar el aprendizaje, no solo del-la alumno-a sino del-la maestro-a como una condición necesaria a la que se debe aspirar con el desarrollo de un portafolios, convierte a la evaluación en una oportunidad de aprendizaje para el sujeto que aprende y para el que enseña. De modo que tomar consciencia, conocerse a sí mismo y saber cómo uno aprende deben ser aspiraciones de la práctica del portafolios. Es decir, es necesario estimular la metacognición; de acuerdo con Pérez Gómez (2012, p. 210), "la metacognición como medio para promover la capacidad de autonomía y autorregulación del aprendizaje y del desarrollo". Wenger (2010), citado por Pérez Gómez (2012), propone considerar tres aspectos fundamentales del diseño como autorregulación que cobran mucho sentido en el desarrollo de un portafolios educativo: una buena orientación, una buena reflexión y una buena exploración de las nuevas posibilidades.

También la autoevaluación, según Pérez Gómez, Soto Gómez, Sola Fernández y Serván (2009), es un motor potente para desarrollar la metacognición: la autoevaluación como mecanismo de aprendizaje que implica organizarse, planificarse, reflexionar, etcétera.

En este mismo sentido, la organización del aprendizaje y para el aprendizaje es otra de las claves. Ordenar el trabajo que se va haciendo contribuye a organizar el pensamiento, a reconocer lo que se lleva trabajado y lo que queda por trabajar.

La organización cobra protagonismo en la medida en que el portafolios debe tratar de presentar, representar el proceso de trabajo, la construcción del conocimiento, las idas y venidas del-la estudiante.

En definitiva, el portafolios asume la condición de estar atento al proceso y al contexto en el que se produce el aprendizaje, en tanto en cuanto debe favorecer la reflexión, la autoevaluación, la metacognición, la organización y la autorregulación del conocimiento que se construye sobre el mundo y sobre uno-a mismo-a. En este sentido, podemos definir al portafolios como la trastienda del aprendizaje. 


\subsection{El portafolios debe fomentar un clima de libertad que propicie la comunicación y el aprendizaje auténticos}

Que el-la estudiante asuma responsabilidades debe ser cualidad de una buena práctica con portafolios. Para ello se necesita un clima de libertad donde la autonomía de quien está aprendiendo vaya creciendo gradualmente. Autonomía del-la alumno-a combinada con guía del-la docente. El-la estudiante debe poder decidir, lo cual pasa por crear parcelas de libertad, de negociación, de argumentación y diálogo, alejadas de intenciones que pretendan oprimir, castigar, silenciar o humillar, más propias de la calificación que de ninguna otra práctica de la escuela.

El portafolios que aspira a evaluar debe impregnar el aula de confianza, liberándola de amenazas, sustituyendo el poder que otorga la calificación por la autoridad de la que nos dota la evaluación. Es así como lograremos poco a poco que tengan lugar una comunicación y un aprendizaje auténticos.

Al referirnos a la condición de autenticidad de la comunicación, hablamos de que el portafolios como práctica de evaluación debe dirigir todos sus esfuerzos a que la información que nos da el alumnado sea lo más sincera posible. Asimismo, el portafolios que sirve al aprendizaje implica la comunicación fluida, relevante y veraz entre docente y estudiante. Cobrando especial importancia el papel del-la docente que debe presentarse a los-as alumnos-as como un guía permanente que propicie confianza y seguridad (Gallardo, Sierra, Barquín y Medeiros, 2016). Tal y como acentúan Calero y Calero (2008), el uso del portafolios desde una perspectiva vigostkiana del aprendizaje refuerza el papel del-la maestro-a como figura mediadora, guía, modeladora y facilitadora del aprendizaje y del contexto que lo fomente.

Del mismo modo, el desplazamiento de la calificación, la no constante presencia de la misma en la vida del aula, contribuye a recuperar el tan maltrecho valor de uso del conocimiento que en tantas ocasiones ha sido relegado por el valor de cambio. Trabajar con portafolios en las aulas no debería implicar la presencia continua de pequeñas notas que van obteniendo cada una de las actuaciones del-la estudiante, que fragmentan el aprendizaje en pequeños "logros". Al contrario, debe ser una herramienta de aprendizaje a largo plazo, que ponga al alumnado en situación de construir y reconstruir su trayectoria en el proceso de aprender, con la mediación del-a profesor-a, y respetuosa con los ritmos de maduración de cada alumno-a. Se aboga así por un aprendizaje más auténtico, no tan mediatizado por las recompensas de la calificación.

Por tanto, el portafolios no debe ser -como no debe serlo el conocimiento- una mera mercancía sin ninguna utilidad más allá de recopilar los "logros" para ser expuestos y valorados por el profesorado y, de este modo, intercambiados por una calificación.

\subsection{El portafolios debe apostar por el error como fuente de aprendizaje y desplazar los juicios penalizadores}

Una de las condiciones de la evaluación es la de preocuparse por los errores del alumnado, no en el sentido de penalizarlos, sino en el sentido de comprenderlos y transformarlos. Como exponen Murillo e Hidalgo (2015), en lugar de ser un mecanismo de control y castigo, la evaluación debe centrarse en reforzar lo positivo y en servir de ayuda al alumnado. Debemos practicar, en palabras de estos autores, una "evaluación optimista". También Postman (2001) reconoce que la historia del aprendizaje es la narración de nuestros errores superados. Por tanto, el portafolios debe servir para buscar las respuestas a los problemas que el alumnado se va encontrando a 
lo largo de su proceso de aprendizaje, así como para solucionar los errores cometidos y sobreponerse a las adversidades.

El portafolios debe ayudar al alumnado a construir una mente informada y organizada de lo que aprende y cómo lo aprende. En este sentido, cobra relevancia la corrección informada, es decir, comentarios que orienten a quien está en situación de aprendizaje, ayudándole a tomar consciencia de sus puntos fuertes y sus puntos débiles y de cómo fortalecer estos últimos.

En definitiva, la corrección informada es, en gran parte, la antesala a la reflexión de la que hablábamos anteriormente.

\subsection{El portafolios debe ser un canal que recoja la voz del alumnado y ayude a la comprensión holística de la vida del aula}

El portafolios, para que sea útil a la evaluación, debe permanecer atento a la voz y al sentir del alumnado. Es sin duda el-la alumno-a quien construye su aprendizaje y por tanto quien va dando forma y sentido al proceso de creación del portafolios. En este sentido, está bastante claro que el portafolios tiene que dar voz al-la estudiante, porque es "su" voz la que debe sonar. Pero reconocer la voz y la participación del alumnado en el aula no es solo escuchar lo que tiene que decir sobre sí mismo-a, sobre su propio proceso; es animarle a que exprese también cuestiones sobre el contexto que rodea a ese proceso.

Los-as alumnos-as tienen opiniones sobre las condiciones en las que tiene lugar la enseñanza y el aprendizaje; tienen opiniones de los-as docentes, de los materiales, de las actividades, de los tiempos, de los agrupamientos, del clima de clase, entre otras. Unas veces más sustanciosas y otras menos. Ahí es donde juega un papel fundamental el portafolios, que debe fomentar la participación activa en el aula. El-la maestro-a que se atreve con un portafolios debe atreverse con la democracia. Preguntar, escuchar, debatir, dialogar, argumentar, negociar cualquier aspecto relacionado con la vida escolar, es también trabajar con portafolios. Este debe ser un ejercicio de alimentar la palabra escrita pero también la palabra hablada. El portafolios al servicio de la evaluación es un ejercicio democrático.

Para ello, los-as docentes deben abrir vías de comunicación constantes que recojan manifestaciones orales, escritas, anónimas, nominales, individuales y grupales sobre los variados elementos que confluyen en el día a día del aula, no solo dando la palabra al-la alumno-a, sino ayudándole a expresarla y a utilizarla; lo cual requiere dedicación, tiempo y paciencia.

\subsection{El portafolios debe convertirse en un sistema de evaluación}

Llegados a este punto, tras haber expuesto todo lo que el portafolios debe ser a la vez, se hace necesario resaltar la necesidad de convertir a este en un sistema de evaluación compuesto por diferentes herramientas que conectadas entre sí deben transformar la práctica educativa del aula.

El portafolios no es un medio físico a través del cual el alumnado presenta su trabajo y el profesorado lo valora. Para que realmente este sirva a la evaluación y por tanto al aprendizaje, debe ser una manera de entender la práctica educativa, una forma de vida en el aula, un sistema complejo que pretenda abarcar todos los elementos influyentes en el proceso de enseñanza-aprendizaje y que trate de arrojar luz sobre todos ellos. 
Pérez Gómez y Serván (2016) defienden la idea del portafolios no como instrumento de evaluación sino como sistema que puede contener otros instrumentos y recursos que han de coordinarse.

Para que realmente el portafolios tenga sentido educativo no debería ser reducido a la categoría de instrumento, sino abrirse, complejizarse en el sentido de incluir un conjunto de herramientas y estrategias puestas en práctica con una finalidad: la de comprender y mejorar el proceso de enseñanza-aprendizaje. Para ello, es necesario incorporar una serie de elementos que nos permitan abarcar y analizar de forma holística la realidad del aula. Alcaraz, Mayorga y Pérez (2016) exponen la importancia de incluir en los portafolios educativos material de anclaje elaborado por el-la docente, como tablas, fichas, cuestionarios, que sirvan de guía al alumnado para preguntarse y reflexionar sobre el proceso en el que se encuentra, sobre cómo ha llegado hasta ahí y cómo ha de representarlo. Veremos a continuación algunas sugerencias de herramientas y estrategias que pueden integrar la práctica de evaluar a través de portafolios:

$\checkmark$ Diarios: A través de los diarios el profesorado puede ir promoviendo gradualmente el nivel de reflexión del alumnado que suele estar poco habituado a ello. A medida que los-as estudiantes trabajan en solitario o en grupo, construir un diario individual o colectivo puede resultar una oportunidad para que estos-as vayan incorporando poco a poco elementos de análisis sobre lo que aprenden y cómo lo aprenden. Es importante aquí no desesperar si la calidad de las primeras reflexiones es baja y que el-la docente se configure como un elemento de apoyo en ese camino para que poco a poco el nivel de profundidad y argumentación vaya creciendo.

$\checkmark$ Cuestionarios de autoevaluación: Plantear al alumnado preguntas sobre cuestiones relativas a su propio proceso de aprendizaje puede resultar útil para que poco a poco este vaya tomando consciencia de lo que ha trabajado, de lo que le ha resultado más o menos difícil, de lo que más le ha gustado, de lo que le queda por mejorar, de los compromisos que adquiere, etcétera. Incorpora la valoración cualitativa que el-la alumno-a hace de su trabajo. Las preguntas pueden empezar siendo sencillas y, a medida que se va avanzando, estas pueden requerir respuestas algo más elaboradas. El cuestionario de autoevaluación es un recurso útil para el sujeto que aprende y para quien enseña, puesto que de este modo el-la profesor-a "accede" a lo que el-la alumno-a piensa sobre su proceso de aprendizaje. Para que este tenga sentido es necesario demostrar al alumnado que no se trata de auto-ponerse nota y que ello no tiene implicaciones en la calificación final ya que, si no, es muy probable que este responda en función de lo que cree que queremos oír. Es recomendable que no sea anónimo para que de este modo el-la docente pueda detectar necesidades.

$\checkmark$ Fichas u hojas de registro: Se trata de poner al alumnado en situaciones en las que deba ir anotando el trabajo que se lleva hecho. Es el lugar (puede ser una tabla compuesta por filas y columnas) donde se registran los proyectos realizados, las actividades desarrolladas, etcétera, de forma sintética. Para que tenga sentido educativo, puede ir acompañada de observaciones tanto del-la estudiante como del-la profesor; de este modo, se da lugar a una especie de diálogo entre las partes ya que el-la alumno-a registra un comentario acerca de un proyecto o actividad y el-la docente puede contestarle por escrito. 
$\checkmark$ Cuestionarios de evaluación: Se trata de un listado de preguntas que el-la docente hace al alumnado sobre aspectos generales o concretos del desarrollo de clase. Debe contestarse de forma anónima dado que el sentido de este es conocer el punto de vista del alumnado, la opinión que este construye acerca del modo en que se trabaja, del clima de clase o de la actitud del-a profesor-a. Para ello, es importante crear un clima de confianza y seguridad en el que los-as estudiantes puedan expresarse con libertad. Se trata de incorporar la voz del alumnado sobre lo que acontece en el aula, de escucharle e incorporar a nuestro quehacer diario el modo en que los protagonistas del mismo perciben y entienden nuestras actuaciones.

$\checkmark$ Buzones de Freinet: Son los conocidos buzones "propongo", "felicito" y "critico", cuya finalidad es animar al alumnado a dejar mensajes a compañeros-as o maestros-as del aula, incluso del centro. Felicitar a alguien, criticar una acción o proponer algo es otro modo de formar parte activa de la vida escolar. El mecanismo es sencillo: los buzones deben permanecer fijos en el aula y disponibles para ser usados en cualquier momento. Se debe planificar un día (puede ser uno a la semana o cada quince días) para la lectura de los mensajes. Como todas las anteriores, se trata de una práctica que hay que trabajar y alimentar dentro de un clima de seguridad, confianza y afecto.

$\checkmark$ Corrección de las producciones del alumnado: Se trata de incorporar al portafolios las producciones del alumnado, con objeto de ser leídas y corregidas por el-la docente que debe proporcionar retroalimentación a sus autores-as, de modo que estos-as puedan reconducir el trabajo, incorporar nuevas cuestiones, ampliar o reducir, atendiendo a las sugerencias del-la profesor-a.

En definitiva, destacamos que tal y como afirman Calero y Calero (2008):

Entendemos que el valor real del portafolios que planteamos no reside en su apariencia física o en su organización concreta, sino en el contexto que crea de desarrollo y de cooperación que generan los procesos de reflexión y colaboración entre profesor y alumno. (p.32)

\section{Concluyendo}

A lo largo de este artículo se han expuesto no solo el origen de las prácticas con portafolios en el ámbito educativo como herramienta de evaluación, sino algunos de los diferentes tipos y usos que se desprenden de dicha práctica. A su vez, en este trabajo se han descrito una serie de condiciones que un portafolios al servicio de la evaluación debería cumplir. Entendiendo la evaluación como proceso de recogida de información, planificado y horizontal, que no solo recoge sino que devuelve información que sea útil para conocer, comprender y analizar lo que influye en el proceso de enseñanzaaprendizaje, con la intención de mejorarlo (Alcaraz, 2015b).

También se ha resaltado la relativa novedad del uso de portafolios en el ámbito educativo, especialmente en la etapa obligatoria, por lo que es importante destacar que, ahora más que nunca, nos encontramos ante el reto de tratar de dotarlo de sentido educativo, situándolo al servicio de la evaluación. Pero ¿qué significa esto? Significa que, como hemos ido exponiendo, debemos convertirlo en un medio y en un fin para el aprendizaje. En una oportunidad para analizar y reflexionar sobre los procesos y los contextos de aprendizaje; en un medio para propiciar un clima de libertad, participación y comunicación auténtica. En una vía donde el error sea considerado fuente de 
aprendizaje. Es decir, en un sistema, compuesto a su vez por otras herramientas o instrumentos que obedezcan a una filosofía concreta de entender y vivir la evaluación en el aula. Descartando la función sumativa y resaltando la formativa, ya que podemos correr el riesgo de trasladar el uso de rendición de cuentas que del portafolios se ha empezado a hacer en contextos de educación superior, donde la función selectiva del sistema es mucho más clara y acentuada que en la enseñanza obligatoria.

De sobra es conocido que no son las herramientas en sí mismas las que limitan o posibilitan mejores o peores condiciones de aprendizaje, sino que son sus usos. Usos que dependen directamente de las concepciones de aprendizaje, enseñanza, currículum y evaluación de la persona que las pone en práctica. De modo que cualquier herramienta puede estar al servicio de la calificación o al de la evaluación. Así, por ejemplo, el examen podría estar al servicio de una u otra según cómo se use y para qué se use. Sin embargo, y aunque es probablemente cierto que lo que conocemos por examen pueda emplearse de una forma u otra en función de quién lo emplea, ocurre particularmente con el examen que, al ser una herramienta con tan larga trayectoria calificativa, la línea entre utilizarlo para calificar y evaluar es sumamente fina. Por este motivo, insistimos en la crítica hacia los 'usos' y 'desusos' del examen como herramienta de calificación y/o evaluación ya que, si queremos contribuir a ofrecer claridad al debate evaluación-calificación, apostar por la evaluación sin desprendernos de los exámenes -pesos pesados de la calificación-, es arriesgarnos a seguir mezclando todo.

Por eso, insistimos en que una de las ventajas del portafolios es precisamente su novedad en el ámbito de la pedagogía; cualidad que no lo exime de posibles riesgos, tal y como apuntábamos antes (la trayectoria en otros contextos como repositorio que "demuestra" nuestros méritos o capacidades). Por lo tanto, es importante que la cualidad continua del desarrollo de un portafolios para evaluar en el aula no se convierta en la barita mágica con la que tratemos de "medir" constantemente las actitudes, acciones, manifestaciones, etcétera, del alumnado. Como plantea Pérez Gómez (2012, p. 96): "Las recomendaciones de la evaluación continua se han malentendido o convertido en exigencias de examen o calificación continua”.

En definitiva, podemos decir que entre la ventaja y el riesgo asumimos un reto: aprovechar el carácter continuo y reflexivo que se le otorga en la literatura al portafolios para convertirlo en una oportunidad de evaluación "para" el aprendizaje, más que "sobre" el aprendizaje, ya que caer en la tentación de usar el portafolios para puntuar constantemente lo que los-as estudiantes realizan en clase sería desperdiciar una buena ocasión para plantear en el aula una auténtica evaluación .

\section{Referencias}

Alcaraz, N. (2014). Un viejo trío de conceptos: Aprendizaje, currículum y evaluación. Aula De Encuentro, 2(16), 55-86.

Alcaraz, N. (2015a). Aproximación histórica a la evaluación educativa: De la generación de la medición a la generación ecléctica. Revista Iberoamericana de Evaluación Educativa, 8(1), 11-25.

Alcaraz, N. (2015b). Evaluación vs. calificación. Aula de Encuentro, 17(2), 209-236. 
Alcaraz, N., Fernández, M. y Sola, M. (2012). La voz del alumnado en los procesos de evaluación docente universitaria. Revista Iberoamericana de Evaluación Educativa, 5(2), 2739.

Álvarez Méndez, J. M. (2001). Evaluar para conocer, examinar para excluir. Madrid: Morata.

Álvarez Méndez, J. M. (2008). Evaluar el aprendizaje en una enseñanza centrada en las competencias. En J. Gimeno Sacristán (Comp.), Educar por competencias, ¿̨qué hay de nuevo? (pp. 206-234). Madrid: Morata.

Barberá, E. (2005). La evaluación de competencias complejas: La práctica del portafolio. Educere, 9(31), 497-503.

Barragán, R. (2005). El portafolio, metodología de evaluación y aprendizaje de cara al nuevo Espacio Europeo de Educación Superior. Una experiencia práctica en la Universidad de Sevilla. Revista Latinoamericana de Tecnología Educativa, 4(1), 121-139.

Barret, H. (2001). Electronic portfolios. Educational technology. En Educational Technology Enciclopedia, ABC-CLIO. Recuperado de http://electronicportfolios.com/portfolios/encyclopedaientry.htm

Barret, H. (2010). Balancing the two faces of eportfolios. Educação, Formação y Tecnologías, 3(1), 6-14.

Bastidas, J. A. (1996). The teaching portfolio. A tool to become a reflective teacher. English Teaching Forum, 34(3-4), 24-48.

Calero, A. y Calero, E. (2008). El portfolio como estrategia de evaluación compartida de la comprensión lectora. Didáctica. Lengua y Literatura, 20, 15-36.

Calfee, R. C. y Freedman, S. W. (1996). Classroom writing portfolios: Old, new, borrowed, blue. En R. Calfee y P. Perfumo (Eds.), Writing portfolios in the classroom (pp. 3-26). Mahwah, NJ: Lawrence Erlbaum Associates.

Danyelson, C. y Abrutyn, L. (1999). Una introducción al uso del portafolios en el aula. México D. F.: Fondo de Cultura Económica.

Darling-Hammond, L. (2005). Standards and assessments: Where we are and what we need. Teachers College Record. The Voice of Scholarship in Education. Recuperado de http://www.tcrecord.org/PrintContent.asp?ContentID=11109

Delmastro, A. L. (2005). El uso del portafolios en la enseñanza de las lenguas extranjeras. Perspectiva del docente. Investigación y Postgrado, 20(2), 187-211.

Díaz Barriga, F. (2006). Enseñanza situada: Vinculo entre la escuela y la vida. México D. F.: McGraw- Hill.

Díaz Barriga, F. y Pérez, M. (2010). El portafolio docente a escrutinio: Sus posibilidades y restricciones en la formación y evaluación del profesorado. Observar, 4, 6-27.

French, R. L. (1992). Portfolio assessments and LEP students. En T. D'Emilio y S. Coleman (Coord.), Focus on evaluation and measurement: Proceedings of the Second National Research Symposium on Limited English Proficient Student Issues (pp. 249-285). Washington D. C.: United States Department of Education, OBEMLA.

Gallardo, M., Sierra, E., Barquín, F. J. y Medeiros, M. (2016). Despertando las miradas: Propósitos, criterios y sentido del portafolios educativo. En A. I. Pérez Gómez (Dir.), El portafolios educativo en Educación Superior (pp. 65-78). Madrid: Akal.

García Hernández, E. (2000). Algunas aplicaciones del portafolios en el ámbito educativo. Chihuahua: Secretaría de Educación y Cultura del Estado de Chihuahua. Recuperado de http://sgpwe.izt.uam.mx/files/users/virtuami/file/ext/gestion_evaluacion_actv_ext_p ortafolios.pdf 
Hamilton, S. J. (1994). Portfolio pedagogy: Is a theoretical construct good enough? En L. Black, D. A. Daiker, J. Sommers y G. Stygall (Eds.), New directions in portfolio assessment (pp. 157-167). Portsmouth, NH: Boynton/Cook Publishers, Heinemann.

Hebert, E. (2001). The power of portfolios. What children can teach us about learning and assessment. San Francisco, CA: Jossey-Bass.

Hernández Hernández, F. (2008). El portafolios electrónico y el aprendizaje para la comprensión en Secundaria. Investigación en la Escuela , 64, 59-71.

Hidalgo, J. y De la Blanca, S. (2013). La contribución del prácticum a la innovación metodológica en la educación de adultos. En P. C. Muñoz Carril, M. Raposo Rivas, M. Gónzalez Sanmamed, M. E. Martínez Figueira, M. A. Zabalza Cerdeiriña y A. Pérez Abellás (2013), Un prácticum para la formación integral de los estudiantes (pp. 943-953). Santiago de Compostela: Andavira.

Jalbert, P. (1998). Le portfolio au service de l'apprentissage et de l'évaluation. Montréal-Toronto: Chenelière/McGraw-Hill.

Klenowski, V. (2004). Desarrollo del portafolios para el aprendizaje y la evaluación. Procesos y principios. Madrid: Narcea.

Le Boterf, G. (2000). Construire les compétences individuelles et collectives. París: Les Editions d'Organisation.

Lyons, N. (Comp.). (1999). El uso de portafolios. Propuestas para un nuevo profesionalismo docente. Buenos Aires: Amorrortu.

Meeus, P. y Lois, V. (2006). Portfolio in higher education: Time for clarifactory framework. International Journal of Teaching and Learning in Higher Education, 17(2), 127-135.

Monereo, C. (2009). La autenticidad de la evaluación. En M. Castelló (Coord.), La evaluación auténtica en Enseñanza Secundaria y Universitaria (pp. 9-22). Barcelona: Edebé.

Murillo, F. J. e Hidalgo, N. (2015). Dime cómo evalúas y te diré qué sociedad construyes. Revista Iberoamericana de Evaluación Educativa, 8(1), 5-9.

Murphy, S. y Camp, R. (1996). Moving toward systemic coherence: A discussion of conflicting perspectives on portfolio assessment. En R. Calfee y P. Perfumo (Eds.), Writing portfolios in the classroom (pp. 103-148). Mahwah, NJ: Lawrence Erlbaum Associates.

Oosterhof, A. (1996). Classroom applications of educational measurement. Columbus, OH: Merrill.

Paulson, F., Paulson, P. y Meyer, C. (1991). What makes a portfolio a portfolio. Educational Leadership, 60-63.

Pelavin, S. (1991). Performance assessments in the states. Washington, DC: Pelavin Associates.

Pérez Gómez, A. I. (2012). Educarse en la era digital. Madrid: Morata.

Pérez Gómez, A. I., Soto Gómez, E., Sola Fernández, M. y Serván, M. J. (2009). La evaluación como aprendizaje. ¿̇valuación = Calificación? Madrid: Akal.

Pérez Gómez, A. I. y Serván, M. J. (2016). Naturaleza y sentido del portafolios educativo. En A. I. Pérez Gómez (Dir.), El portafolios educativo en Educación Superior (pp. 39-45). Madrid: Akal.

Pérez, L., Alcaraz, N. y Fernández, M. (2016). Revisión bibliográfica sobre el portafolios. En A. I. Pérez Gómez (Dir.), El portafolios educativo en Educación Superior (pp. 17-38). Madrid: Akal.

Pérez Rendón, M. (2014). Evaluación de competencias mediante portafolios. Perspectiva Educacional, 53(1), 19-35. doi:10.4151/07189729-Vol.53-Iss.1-Art.213 
Pollari, P. (2000). This is my portfolio: Portfolios in upper secondary school English studies. Recuperado de http://eric.ed.gov/PDFS/ED450415.pdf

Postman, N. (2001). El fin de la educación. Una nueva definición del valor de la escuela. Barcelona: Octaedro.

Reckase, M. (1995). Portfolio assessment: A theoretical estimate of score reliability. Educational Measurement: Issues and Practice, 14(1), 12-14. doi:10.1111/j.1745-3992.1995.tbo0846.x

Román, M. y Murillo, F. J. (2009). La evaluación de los aprendizajes escolares. Un recurso estratégico para mejorar la calidad educativa. Revista Iberoamericana de Evaluación Educativa, 1(2), 5-9.

Shores, E. F. y Grace, C. (2004). El portafolios paso a paso: Infantil y primaria. Barcelona: Graó.

Soto, E. (2016). La reflexión corazón y alma del portafolios educativo. En A. I. Pérez Gómez (Dir.), El portafolios educativo en Educación Superior (pp. 97-118). Madrid: Akal.

Starkie, E. (2009). El papel del docente y del alumno en la elaboración de portafolios. Revista de Educación, 349, 451-462.

Villalobos, J. (2002). Portafolios y reflexión: Instrumentos de evaluación en una clase de escritura. Educere, 5(16), 390-396.

Wenger, E., White, N. y Smith, J. (2010). Digital habitats, stewarding technology for comunities. Portland, OR: CPsquare.

Wiggings, G. (1990). The case for authentic assessment. Recuperado de http://www.ericdigests.org/pre-9218/case.htm

Wile, J. M. y Tierney, R. J. (1996). Tensions in assessment: The battle over portfolios, curriculum and control. En R. Calfee y P. Perfumo (Eds.), Writing portfolios in the classroom (pp. 203-215). Mahwah, NJ: Lawrence Erlbaum Associates. 